\title{
GRAM MATRICES OF REPRODUCING KERNEL HILBERT SPACES OVER GRAPHS III
}

\author{
Michio SETO AND SHO SUdA
}

Abstract. We study reproducing kernel Hilbert spaces induced by inclusion $G_{1} \subset G_{2}$ of two connected graphs having a common vertex set. Under a certain finiteness condition, it is shown that the dimensions of de Branges-Rovnyak complements associated with inclusion $G_{1} \subset G_{2}$ are described by the language of graph theory.

Mathematics subject classification (2010): Primary 47B32, Secondary 05C50.

Keywords and phrases: Reproducing kernel Hilbert space, graph, de Branges-Rovnyak space.

\section{REFERENCES}

[1] B. BollobÁs, Modern graph theory, Graduate Texts in Mathematics 184, Springer-Verlag, New York, 1998.

[2] J. A. BALl AND V. Bolotnikov, de Branges-Rovnyak spaces: basics and theory, arXiv:1405.2980v1 [math.CA], 12 May 2014.

[3] P. G. Doyle And J. L. SNEll, Random walks and electric networks, Carus Mathematical Monographs, 22, Mathematical Association of America, Washington, DC, 1984.

[4] P. E. T. JORGENSEN AND F. TIAN, Discrete reproducing kernel Hilbert spaces: sampling and distribution of Dirac-masses, J. Mach. Learn. Res. 16 (2015), 3079-3114.

[5] P. E. T. Jorgensen And F. Tian, Frames and factorization of graph Laplacians, Opuscula Math. 35, 3 (2015), 293-332.

[6] P. E. T. Jorgensen and E. P. J. Pearse, A Hilbert space approach to effective resistance metric, Complex Anal. Oper. Theory 4, 4 (2010), 975-1013.

[7] D. SARASON, Sub-Hardy Hilbert spaces in the unit disk, University of Arkansas, Lecture Notes in the Mathematical Sciences 10, A Wiley-Interscience Publication, John Wiley \& Sons, Inc., New York, 1994.

[8] M. SETo, Composition operators induced by injective homomorphisms on infinite weighted graphs, J. Math. Anal. Appl. 435, 2 (2016), 1467-1477.

[9] M. Seto, S. Suda And T. TANiguchi, Gram matrices of reproducing kernel Hilbert spaces over graphs, Linear Algebra Appl. 445 (2014), 56-68.

[10] M. Seto, S. Suda AND T. TANiguchi, Gram matrices of reproducing kernel Hilbert spaces over graphs II (graph homomorphisms and de Branges-Rovnyak spaces), Nihonkai Math. J. 26, 1 (2015), $15-29$.

[11] V. I. VASYUNIN AND N. K. NiKOL'SKIĬ, Quasiorthogonal decompositions with respect to complementary metrics, and estimates of univalent functions, Leningrad Math. J. 2, 4 (1991), 691-764. 